\title{
La industria forestal del Estado de Chihuahua
}

\author{
Laura C. Ruelas Monjardín \\ Raymundo Dávalos Sotelo ${ }^{1}$
}

\section{RESUMEN}

Se presenta un panorama general de la industria forestal del estado de Chihuahua, tomando en cuenta su desarrollo de las últimas tres décadas. Se toma como referencia a su más fuerte competidora, que es la del estado de Durango y el lugar que ambas ocupan en el contexto nacional. Se presentan las estadísticas de la superficie arbolada del país y de las existencias de madera.

La producción forestal del estado ha venido experimentando decrementos año con año; con respecto al total nacional, el sector agropecuario, silvícola y pesquero tiene una aportación importante al PIB. Esta presencia es aún más importante si se considera, que dentro del sector, la industria de la madera y sus derivados aportó el $15 \%$ del PIB de la industria manufacturera. En cuanto a número de industrias establecidas, en la última década el número se mantiene estable.

Se plantean los problemas principales que enfrenta la industria forestal en este Estado y algunas medidas que los sectores gubernamental y privado están tomando para contrarrestar los efectos negativos de la apertura comercial y de la desventaja tecnológica de este sector industrial.

PALABRAS CLAVE:

Industria forestal, Estado de Chihuahua, Estado de Durango.
ABSTRACT

This paper offers an outlook of the forest industry of Chihuahua, taking into account its development over the last three decades; for this purpose it is considered, for comparative ends, its strongest competitor, that of Durango state, and the place that both occupy in the national context. Statistical data are presented in regards to the forest surface and the data on wood stocks.

The state forest production has being experiencing yearly decrements. With respect to the national context, the farming and animal husbandry, forestry and fishery sector of Chihuahua has an outstanding support of the Gross Domestic Product (GDP). This presence is stronger if it is considered that the wood and wood based industry provided $15 \%$ of the GDP of the manufacturer industry. In terms of the number of established industries, in the last decade, this number keeps stable.

The main problems that the state forestry industry face are presented, along with some measures that the goverment and the private sector are taking in order to offset the negative impact of the commercial globalization and the technological lag that this sector currently show.

KEY WORDS:

Forestry industry, State of Chihuahua, State of Durango.

1 Departamento de Productos Forestales y Conservación de Bosques. Instituto de Ecología, A.C. Apdo. Postal 63. Xalapa, Ver. 91000 México.

Manuscrito recibido para su publicación el 7 de abril de 1999. 


\section{INTRODUCCION}

El estado de Chihuahua, abarca una superficie de 24,814,069 ha. Está dividido políticamente en 67 municipios, tiene una población de 2,792,989 habitantes (al 5 de noviembre de 1995) y una baja densidad de población rural $\left(2 \mathrm{hab} / \mathrm{km}^{2}\right)$. El $71 \%$ del territorio estatal se considera apto para actividades forestales. Chihuahua es el estado con mayor superficie arbolada, ya que posee $7,591,842$ ha, $13.2 \%$ del total nacional (Tabla 1); esta superficie se extiende de norte a sur en una franja de 850 $\mathrm{km}$ desde el municipio de Janos hasta el de Guadalupe y Calvo, e incluye un total de 30 municipios. Sin embargo, en cuanto a existencias de madera, ocupa el segundo lugar a nivel nacional, con el $14 . \%$ del total, ya que dispone de $266,112,404 \mathrm{~m}^{3}$ con un incremento corriente anual de 4 millones de $\mathrm{m}^{3}$ (CNIF, 1991). Sólo es superado por Durango. También es el segundo productor de madera (CNIF, 1994) (Tabla 2).

En la entidad, especial importancia tiene la región de la Tarahumara, ya que constituye una de las principales reservas de recursos naturales del estado. Ocupa una superficie aproximada de $65,000 \mathrm{~km}^{2}$.
Esta región, que se ubica al suroeste del estado, comprende 23 municipios (SEMARNAP, 1999). Del total de 3,568,663 ha de superficie de predios forestales, aproximadamente, el $80 \%$ se ubica en esta región. De los bosques en aprovechamiento un $82 \%$ es de propiedad ejidal, sumando 186 predios; el $6 \%$ es de propiedad comunal con 22 predios; el $3 \%$ es de propiedad colonial con sólo 5 predios, y se tienen 450 predios particulares en aprovechamiento, que representan el $9 \%$ de la superficie (SPE, 1999).

\section{VEGETACION Y CLIMA}

Su vegetación incluye 5 ecosistemas básicos: bosque de clima templado frío, selvas de clima subtropical y tropical, vegetación de zona árida, vegetación halófila y áreas perturbadas (SARH, 1994). De manera mayoritaria pertenece al ecosistema templado-frío y constituye el pilar de la industria forestal mexicana, ya que más del $60 \%$ de las especies de pino tienen importancia comercial y el $80 \%$ de los productos forestales del país se obtienen de los bosques de pino-encino.

Tabla 1. Area forestal nacional y de los estados de Chihuahua y Durango (ha)

\begin{tabular}{||l|l|c|c|c||}
\hline \hline ESTADO & CHIHUAHUA & DURANGO & NACIONAL \\
\hline \multirow{2}{*}{$\begin{array}{l}\text { Superficie } \\
\text { arbolada }\end{array}$} & Bosques (ha) & $7,086,591$ & $4,989,401$ & $30,433,893$ \\
\cline { 2 - 5 } & Selvas (ha) & 505,251 & 495,020 & $26,440,061$ \\
\cline { 2 - 5 } & Total arbolado & $7,591,842$ & $5,484,421$ & $56,873,954$ \\
\hline $\begin{array}{l}\text { Otras áreas } \\
\text { forestales }\end{array}$ & $\begin{array}{l}\text { Vegetación de zonas } \\
\text { áridas (ha) }\end{array}$ & $8,686,466$ & $2,671,571$ & $58,472,398$ \\
\cline { 2 - 5 } & $\begin{array}{l}\text { Vegetación Hidrófila } \\
\text { y Halófila (ha) }\end{array}$ & 480,996 & 100,815 & $4,163,343$ \\
\cline { 2 - 5 } & $\begin{array}{l}\text { Areas perturbadas } \\
\text { (ha) }\end{array}$ & 768,527 & 872,094 & $22,235,474$ \\
\cline { 2 - 5 } & $\begin{array}{l}\text { Total otras áreas } \\
\text { (ha) }\end{array}$ & $9,935,989$ & $3,644,480$ & $84,871,215$ \\
\hline \multicolumn{2}{|l|}{ Total Forestal } & $17,527,831$ & $9,125,901$ & $141,745,169$ \\
\hline
\end{tabular}

Fuente: SARH, 1994. 
Tabla 2. Principales estados productores de madera, 1995

\begin{tabular}{||l|c|c|c|c||}
\hline \multirow{2}{*}{} & \multicolumn{2}{|c|}{$\begin{array}{c}\text { Producción de todas } \\
\text { las especies }\end{array}$} & \multicolumn{2}{c|}{$\begin{array}{c}\text { Producción de madera } \\
\text { de pino }\end{array}$} \\
\cline { 2 - 5 } & $\mathrm{m}^{3}$ rollo & $\%$ & $\mathrm{~m}^{3}$ rollo & $\%$ \\
\hline Nacional & $6,302,417$ & 100 & $5,351,723$ & 100 \\
\hline Chihuahua & $1,293,053$ & 21 & $1,188,588$ & 22 \\
\hline Durango & $1,942,737$ & 31 & $1,838,714$ & 34 \\
\hline
\end{tabular}

Fuente: CNIF, 1996.

Dentro de este ecosistema están los tipos de vegetación cuya distribución corresponde en general a la ubicación de las serranías más importantes de México, como son: Sierra Madre Occidental, Sierra Madre del Sur, Sierra Madre Oriental, Sistema Neovolcánico, Macizo de Oaxaca, Sierra Madre de Chiapas, Sierras de Baja California, y otras. Los t i pos de vegetación más representativos del ecosistema son: bosque de pino, bosque de pino-encino, bosque de encino y bosque de otras coníferas.

El clima donde se desarrolla esta vegetación corresponde al templado subhúmedo o semi-seco, con temperatura media anual entre 10 y 20/C, precipitación anual de 600 a $1,000 \mathrm{~mm}$, concentrada de 6 a 7 meses, y en altitudes de entre 1500 y $3000 \mathrm{msnm}$. En estas áreas se presentan heladas todos los años.

Las principales especies que crecen en este ecosistema son:

Pinus arizonica

$P$. duranguensis

P. pseudostrobus

P. patula

P. montezumae

P. teocote

$P$. tenuifolia

Abies religiosa (oyamel)

Cupressus lindleyi (cedro blanco)

Juniperus spp. (táscate)
Libocedrus spp. (cedro)

Quercus spp. (encino)

Alnus spp. (aile)

Arbutus spp. (madroño), y otros

Cabe mencionar que los bosques de abies, Picea y Pseudotsuga en general son de escasa distribución en el país, y se encuentran en algunas localidades de los estados de Durango y Chihuahua.

Algunos de los productos que se extraen de la vegetación de clima templado son: madera aserrada, triplay, productos celulósicos, resinas, mangos de herramientas, parquet, carbón, taninos, etc.

Parte del estado queda comprendido en el ecosistema de zonas áridas (matorral desértico). Las zonas áridas son aquellas cuya precipitación pluvial es menor de 350 $\mathrm{mm}$ al año, con una distribución muy irregular durante la época de lluvias, donde la temperatura media anual varía entre 15/ y $25 / C$ y una época de sequía no menor de 7 meses, con cubierta vegetal generalmente menor al $70 \%$. En las zonas semiáridas, la precipitación anual varía entre 350 a 600 $\mathrm{mm}$ y la cubierta vegetal es mayor del $70 \%$.

Los tipos de vegetación más representativos del ecosistema árido $\mathrm{y}$ semiárido son los siguientes: mezquitales y huizachales; matorral micrófilo, cardonales y tetecheras; izotales, nopaleras y pastizales. 


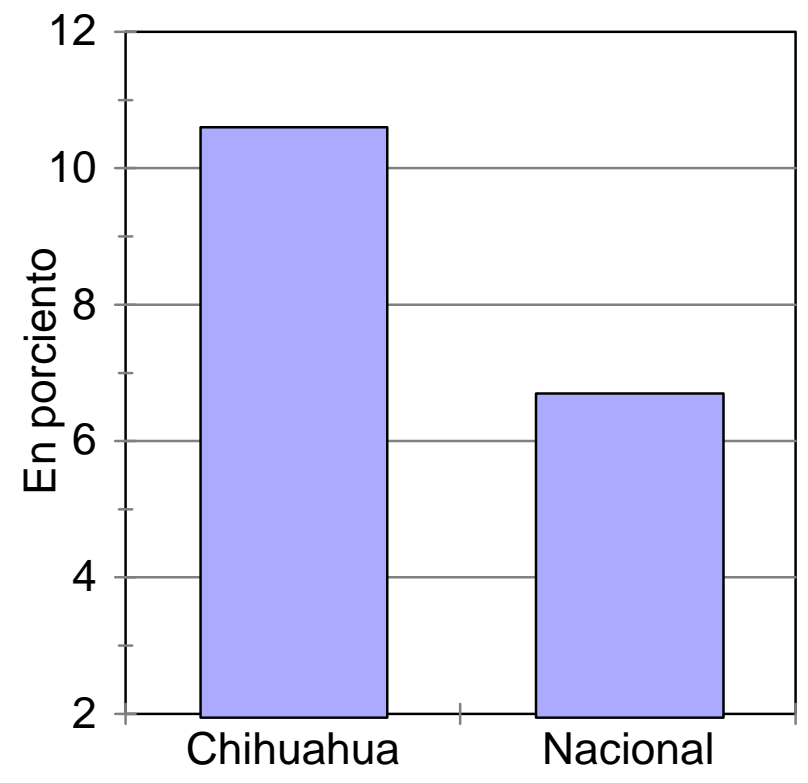

Figura 1. PIB de Chihuahua y Nacional según actividad agropecuaria, silvícola y pesquera, 1993.

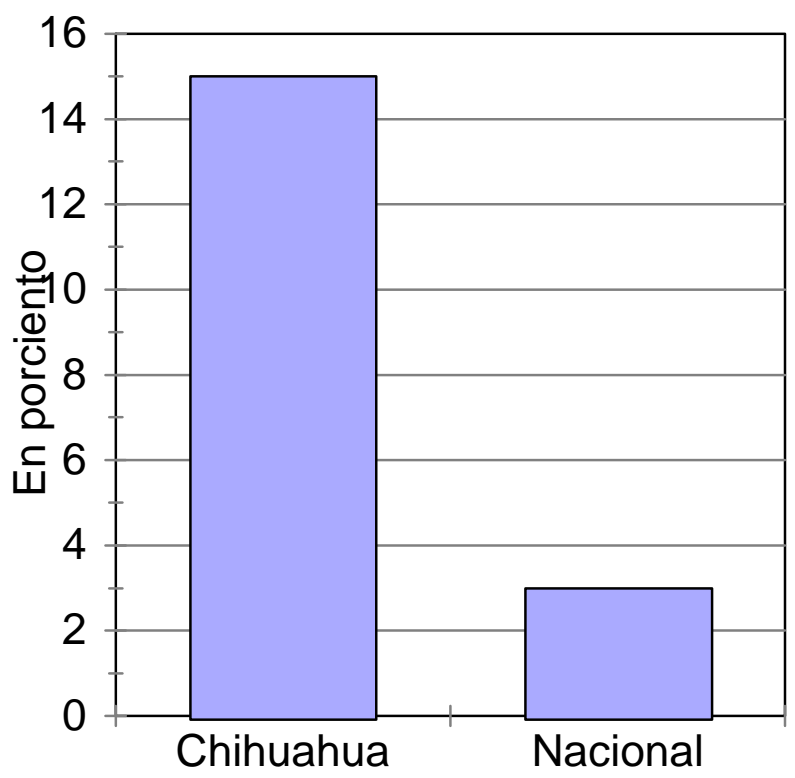

Figura 2. PIB de la industria de la madera y derivados de Chihuahua y Nacional, 1993. 
En general estas áreas se distribuyen al norte del paralelo 21/30' entre las Sierras madre Oriental y Occidental; comprenden parte de los estados de Querétaro, Guanajuato, Aguascalientes, Zacatecas, San Luis Potosí, Durango, Chihuahua, Nuevo León y Coahuila. Asimismo, en los estados de Sonora se encuentra el desierto sonorense y en la Península de Baja California el desierto del mismo nombre (SARH, 1994).

Cabe mencionar que en la entidad, crecen cuatro especies de árboles que se encuentran bajo el estado de conservación, de acuerdo a la revisión que efectuó
SEMARNAP a la norma NOM-059-ECOL1994, estas son: Juglans major, especie amenazada; Picea chihuahuana y Tilia mexicana, en peligro de extinción; y Pinus reflexa, clasificada como especie rara.

\section{EL IMPACTO DE LA INDUSTRIA FORESTAL EN LA ECONOMIA}

El sector agrícola, silvícola y pesquero, y dentro de éste, la industria de la madera y sus derivados tienen un aporte substancial al PIB de la entidad, en comparación con la que éstas tienen en el PIB nacional (Tablas 3, 4, 5 y 6; figuras 1 y 2 : INEGI, 1993).

Tabla 3. Producto Interno Bruto estatal 1970-1993 (miles de pesos)

\begin{tabular}{||l|c|c|c|c|c|c||}
\hline & 1970 & 1975 & 1980 & 1985 & 1988 & 1993 \\
\hline Total estatal & 15,096 & 35,832 & 120,693 & $1,393,319$ & $12,684,878$ & $33,072,552$ \\
\hline $\begin{array}{l}\text { Agropecuario } \\
\text { Silvicultura y Pesca }\end{array}$ & 2,226 & 6,245 & 14,559 & 222,801 & $1,597,007$ & $3,506,096$ \\
\hline
\end{tabular}

Fuente: INEGI, 1993.

Tabla 4. Producto Interno Bruto estatal 1970-1993 (por ciento)

\begin{tabular}{||l|c|c|c|c|c|c||}
\hline & 1970 & 1975 & 1980 & 1985 & 1988 & 1993 \\
\hline Total estatal & 100 & 100 & 100 & 100 & 100 & 100 \\
\hline $\begin{array}{l}\text { Agropecuario, } \\
\text { Silvicultura y Pesca }\end{array}$ & 14.74 & 17.43 & 12.06 & 15.99 & 12.59 & 10.6 \\
\hline
\end{tabular}

Fuente: INEGI, 1993.

Tabla 5. Producto Interno Bruto de la Industria Manufacturera 1970-1993 (miles de pesos)

\begin{tabular}{||l|c|c|c|c|c|c||}
\hline & 1970 & 1975 & 1980 & 1985 & 1988 & 1993 \\
\hline Total estatal & 1,846 & 5,071 & 18,192 & 254,794 & $2,793,125$ & $5,425,066$ \\
\hline $\begin{array}{l}\text { Industria de la Madera y } \\
\text { Productos de la Madera }\end{array}$ & 523 & 1,057 & 5,300 & 61,004 & 594,810 & 811,017 \\
\hline
\end{tabular}

Fuente: INEGI, 1993.

Tabla 6. Producto Interno Bruto de la Industria Manufacturera 1970-1993 (en por ciento)

\begin{tabular}{||l|c|c|c|c|c|c||}
\hline & 1970 & 1975 & 1980 & 1985 & 1988 & 1993 \\
\hline $\begin{array}{l}\text { Industria de la Madera y } \\
\text { Productos de la Madera }\end{array}$ & 28.33 & 20.85 & 29.13 & 23.94 & 21.30 & 14.95 \\
\hline
\end{tabular}

Fuente: INEGI, 1993. 


\section{LA PRODUCCION FORESTAL DE 1991 A 1999}

Para 1991, la producción forestal de Chihuahua representó el $18 \%$ del total nacional; su producción, junto con la de Durango sumaron el $47 \%$ del total nacional. De esta producción la madera de pino ocupa prácticamente el total, con el $99.5 \%$; en un nivel ligeramente inferior se ubica Durango, en donde el pino representó el 96\% de la producción (Tablas 7 y 8 ).

La información obtenida relativa a las extracciones maderables realizadas durante 1991 indican que se produjo un volumen de 7.6 millones de metros cúbicos de madera en rollo, observándose un decremento del $5.5 \%$ con respecto al año inmediato anterior. Este notable retroceso es, sin duda, reflejo de los problemas que aquejan a esta actividad. Si bien para 1991 se observa un retroceso con respecto al año anterior, la producción de madera en 1993 fue de 6.3 millones de metros cúbicos rollo, $17.4 \%$ menos de lo que se produjo en 1992.

De acuerdo con la información de las tablas 9 y 10, se aprecia que para 1997 hubo un descenso en la producción del $7.3 \%$ en comparación con el mismo período de 1996. De esta producción, el pino participó con el $93.6 \%$ de la producción total y su destino fue la escuadría, $65 \%$ en promedio, así como a productos celulósicos, el $30 \%$, y postería el $5 \%$. La producción de encino se destina en su mayoría, más del $90 \%$, a productos celulósicos y el resto a la producción de escuadría. Las otras coníferas se destinan cien por ciento a la producción de postes (SEMARNAP, 1999).
Si se analiza la producción forestal de manera regional, se observa que en la Sierra Madre Occidental, principalmente a lo largo de los Estados de Durango y Chihuahua se extrajo el $46.9 \%$; la segunda región en importancia corresponde a los aprovechamientos realizados en la Sierra Neovolcánica que comprende principalmente los Estados de Jalisco, Michoacán, Veracruz, México y Puebla, produciendo el 31.7\%. En la Sierra Madre del Sur que comprende los Estados de Guerrero y Oaxaca, se extrajo el 9.6\%; el resto de la producción se localiza en otras áreas de clima templado y clima tropical.

Aparentemente para 1996 hubo un descenso radical en el número de industrias forestales, ya que de las 301 establecidas en 1991, para 1996 en el padrón de la Cámara de la Industria Forestal solo se registraron 54 (Tablas 11 y 12). Para el estado de Durango fue mayor la variación, ya que de 391, bajó a 21. Esta información debe tomarse con reservas, debido a dos razones. Una de ellas puede explicarse por el hecho de que después de 1991, la Cámara Nacional de la Industria Forestal dejó de desempeñar la labor de coordinador de las industrias forestales y con ello el acopio de la información. La memoria económica de 1991 fue la más completa que se publicó. Las que se publicaron posteriormente fueron menos específicas. La otra razón, es que en la encuesta aplicada en 1998 por Ruelas (sin publicar) la industria de la impregnación reportó 6 industrias, y de acuerdo con la CNIF, Tabla 12, en 1996 existía una.

Tabla 7. Producción forestal en los estados de Chihuahua y Durango, con respecto al total nacional, 1991 ( $\left.\mathrm{m}^{3} / \mathrm{rollo}\right)$

\begin{tabular}{|l|c|c|c|c|c|c||}
\hline ESTADOS & ESCUADRIA & CELULOSA & $\begin{array}{c}\text { POSTES, PILO- } \\
\text { TES Y MORILLOS }\end{array}$ & $\begin{array}{c}\text { COMBUSTI- } \\
\text { BLES }\end{array}$ & DURMIENTES & TOTAL \\
\hline Ehihuahua & 852,453 & 492,545 & 608 & 696 & 44,495 & $1,390,797$ \\
\hline Purango & $1,779,917$ & 372,279 & 53,752 & 3,227 & 6,686 & $2,215,861$ \\
\hline Nacional & $5,390,964$ & $1,630,635$ & 97,718 & 445,453 & 117,903 & $7,682,673$ \\
\hline
\end{tabular}

Fuente: CNIF, 1991. 
Tabla 8. Producción maderable por especies, 1991 ( $\left.\mathrm{m}^{3} / \mathrm{rollo}\right)$

\begin{tabular}{|l|c|c|c|c|c|c||}
\hline \hline ESTADOS & PINO & $\begin{array}{c}\text { OTRAS } \\
\text { CONIFERAS }\end{array}$ & ENCINO & $\begin{array}{c}\text { OTRAS } \\
\text { LATIFOLIADAS }\end{array}$ & PRECIOSAS & TOTAL \\
\hline Chihuahua & $1,384,126$ & 3,093 & 2,968 & 610 & -- & $1,390,797$ \\
\hline Durango & $2,134,809$ & 24,942 & 51,697 & 1,414 & 2,999 & $2,215,861$ \\
\hline Nacional & $6,437,258$ & 70,721 & 382,777 & 154,035 & 39,174 & $7,682,673$ \\
\hline
\end{tabular}

Fuente: CNIF, 1991.

Tabla 9. Producción de la industria forestal en el estado de Chihuahua, Enero-Agosto 1996-1997 (en m³/rollo)

\begin{tabular}{||l|c|c|c|c|c||}
\hline \multirow{2}{*}{ PRODUCTO } & \multicolumn{2}{|c|}{1996} & \multicolumn{2}{c||}{1997} & $\begin{array}{c}\text { VARIACION } \\
\text { (\%) }\end{array}$ \\
\cline { 2 - 6 } & Volumen & $\%$ & Volumen & $\%$ & $' 97 / 96$ \\
\hline Escuadría & 599,052 & 63.8 & 564,725 & 64.9 & -5.7 \\
\hline Celulósicos & 321,770 & 34.3 & 288,348 & 33.2 & -10.4 \\
\hline Postería & 16,651 & 1.8 & 16,629 & 1.9 & -0.1 \\
\hline Combustibles & 896 & 0.1 & 0 & 0.0 & - \\
\hline Durmientes & & - & 0 & - & - \\
\hline Total & 938,369 & 100.0 & 869,702 & 100.0 & -7.3 \\
\hline \hline
\end{tabular}

Fuente: SEMARNAP, Chihuahua, Subdelegación Forestal y de la Fauna Silvestre, 1999.

Tabla 10. Producción forestal maderable en el estado de Chihuahua, Enero-Agosto 1996-1997 (en m³/rollo)

\begin{tabular}{||l|c|c|c|c|c||}
\hline \multirow{2}{*}{ PRODUCTO } & \multicolumn{2}{|c|}{1996} & \multicolumn{2}{c|}{1997} & Variación (\%) \\
\cline { 2 - 6 } & Volumen & $\%$ & Volumen & $\%$ & '97/'96 \\
\hline Pino & 860,122 & 91.7 & 813,793 & 93.6 & -5.4 \\
\hline Encino & 78,083 & 8.3 & 55,365 & 6.4 & -29.1 \\
\hline $\begin{array}{l}\text { Otras } \\
\text { coníferas }\end{array}$ & 164 & 0.0 & 544 & 0.1 & 231.7 \\
\hline $\begin{array}{l}\text { Otras } \\
\text { latifoliadas }\end{array}$ & 0 & - & 0 & - & - \\
\hline Total & 938,369 & 100.0 & 869,702 & 100.0 & -7.3 \\
\hline
\end{tabular}

Fuente: SEMARNAP, Chihuahua, Subdelegación Forestal y de la Fauna Silvestre, 1999. 
Tabla 11. La participación de Chihuahua en el número de industrias forestales nacionales, 1991

\begin{tabular}{||l|c|c|c|c|c|c|c|c||}
\hline \hline ENTIDAD & $\begin{array}{c}\text { ASERRA- } \\
\text { DEROS }\end{array}$ & $\begin{array}{c}\text { FABRI- } \\
\text { CAS DE } \\
\text { CAJAS }\end{array}$ & $\begin{array}{c}\text { IMPREG- } \\
\text { NADORAS }\end{array}$ & CHAPA & $\begin{array}{c}\text { CONTRA- } \\
\text { CHAPADOS }\end{array}$ & $\begin{array}{c}\text { AGLOME- } \\
\text { RADOS }\end{array}$ & $\begin{array}{c}\text { CELU- } \\
\text { LOSA }\end{array}$ & TOTAL \\
\hline Ehihuahua & 231 & 57 & 4 & --- & 6 & 2 & 1 & 301 \\
\hline Durango & 195 & 177 & 4 & 1 & 10 & 2 & 2 & 391 \\
\hline Nacional & 1543 & 659 & 14 & 18 & 37 & 10 & 17 & 2,623 \\
\hline
\end{tabular}

Fuente: CNIF, 1991

Tabla 12. Padrón de empresas forestales registradas en el Estado de Chihuahua, 1996

\begin{tabular}{|c|c|c|c|c|c|c||}
\hline $\begin{array}{c}\text { ASERRADE } \\
\text { ROS }\end{array}$ & $\begin{array}{c}\text { CARPIN- } \\
\text { TERÍAS }\end{array}$ & $\begin{array}{c}\text { IMPREG- } \\
\text { NADORAS }\end{array}$ & $\begin{array}{c}\text { TABLEROS } \\
\text { Y CHAPAS }\end{array}$ & MADERERÍAS & PLANTACIONES & TOTAL \\
\hline 30 & 9 & 1 & 7 & 6 & 1 & 54 \\
\hline
\end{tabular}

Fuente: CNIF, 1996

Para 1997, la producción forestal maderable continua con un ritmo de desaceleración, ya que si para el periodo $96 / 97$ el descenso fue de $7.3 \%$, en el siguiente lapso, 97/98, fue de $8.9 \%$ (tablas 13 y 14$)$.

La información proporcionada por la Delegación de SEMARNAP en el Estado, indica que para Junio de 1999, 798 predios registraron aprovechamientos, en una superficie total de 3,568,663 ha, de la cual la superficie realmente aprovechada fue $1,237,922$ ha. El volumen total aprovechado fue de 1,984,597 RTA (rollo total árbol). La producción maderable se distribuye para su comercialización en 511 centros de almacenamiento y/o transformación. Estos centros se clasifican de la siguiente manera:

1. Plantas de aserraderos: 279

2. Fábricas de cajas de madera: 85

3. Fábricas de tableros aglomerados: 2

4. Fábricas de triplay: 5

5. Fábrica de celulosa: 1

6. Plantas de impregnación: 6

7. Madererías: 294

Dado que del número total de aserraderos, sólo el $60 \%$ está en operación y que las fábricas de cajas de madera, sólo el $40 \%$ está en operación, el número total de industrias operando ascendería a 217; el resto, 294 estarían representadas por las madererías, cuya función es la compra y venta de madera.

Estos centros de almacenamiento se encuentran bien comunicados a través de autopistas de cuatro carriles, carreteras de dos carriles, la vía del ferrocarril y un gran número de kilómetros de terracería.

Si se analiza el periodo 91-99, el número de industrias forestales, sin contar las madererías, descendió un 30\%, ya que en 1991 existían 301 y para 1999 sólo estaban en operación 217.

\section{LA INDUSTRIA FORESTAL EN EL CONTEXTO INTERNACIONAL}

La apertura comercial mexicana, y en general la dinámica mercantil a nivel mundial, han representado para el subsector forestal una severa reducción de su participación en la economía nacional; entre 1987 y 1994 presentó una reducción de casi el $8 \%$, no obstante que el PIB nacional, en el mismo período, presentó una 
variación positiva de poco más del $21 \%$. De hecho, la contribución anual del subsector forestal al PIB nacional entre 1987 y 1994 , escasamente rebasó el 1\%. (Subcomité Técnico de Plantaciones Forestales, 1995, citado en Alvarez-Icaza, 1997).

A pesar de que México tiene un enorme potencial forestal, en poco más de 56 millones de hectáreas de bosques y selvas, la productividad es hasta cuatro veces menor al promedio mundial; en óptimas condiciones, la producción por hectárea promedio en México, escasamente rebasa un metro cúbico, mientras que en países como Canadá o Estados Unidos es tres veces mayor (Subcomité Técnico de Plantaciones Forestales, 1995, citado en Alvarez-Icaza, 1997).

Derivado de la apertura comercial y de años de estancamiento en el manejo del bosque, la industria forestal nacional enfrenta una severa crisis. Como muestra de lo anterior se puede destacar (Ignacio, 1997):
C Una disminución en la producción forestal maderable del orden del $30 \%$ al pasar de 8.9 millones de $\mathrm{m}^{3}$ en 1989 a 6.3 millones de $\mathrm{m}^{3}$ en 1995, contraria a la tendencia mundial del consumo de madera, que se relaciona principalmente al crecimiento de la población.

C Saldos negativos en la balanza comercial de este sector de hasta $\$ 1,163$ millones de dólares en 1995, lo que representó un incremento del $57 \%$ con respecto a 1989 (Tabla 15).

C Baja en la utilización de las capacidades instaladas de los principales sectores de la actividad industrial forestal.

Entre las causas que dificultan la competencia con los socios del tratado de libre comercio están (Chapela, 1996):

a) La disparidad en los costos de producción, lo que se refleja en los precios de la madera (Tabla 16).

Tabla 13. Producción forestal maderable 1997-1998 (m³/rollo)

\begin{tabular}{||l|c|c|c||}
\hline \hline ESPECIE & 1997 & 1998 & Var. 98/97 \\
\hline Pino & $1,184,167$ & $1,050,720$ & -11.3 \\
Otras coníferas & 54 & 0 & - \\
Encino & 80,084 & 101,597 & 26.9 \\
Otras latifoliadas & 0 & 0 & - \\
Total & $1,284,795$ & $1,152,317$ & -8.9 \\
\hline
\end{tabular}

Fuente: SEMARNAP, 1999

Tabla 14. Producción de la industria forestal 1997-1998 (m³/rollo)

\begin{tabular}{||l|c|c|c||}
\hline PRODUCTO & 1997 & 1998 & Var. 98/97 \\
\hline Escuadría & 824,237 & 762,951 & -7.4 \\
Celulósicos & 416,160 & 375,450 & -9.8 \\
Postería & 24,390 & 13,792 & -43.5 \\
Combustibles & 0 & 124 & - \\
Durmientes & 0 & 0 & - \\
Total & $1,284,795$ & $1,152,317$ & -8.9 \\
\hline
\end{tabular}

Fuente: SEMARNAP, 1999. 
b) Deficiente desarrollo del mercado en México. La inexistencia de un sistema de clasificación no permite la valoración adecuada de la calidad de la madera mexicana; se comercia madera con un sistema de sobredimensiones no cobradas 0 "refuerzo", que pueden llegar a significar pérdidas del orden del $12 \%$ del volumen (Chapela, 1996).

Los productos manufacturados, en general, entraron de inmediato a la libre regulación por el mercado, excepto las tablas de pino, que es uno de los productos más importantes. Aunque los aranceles tratan de otorgar una protección (Tabla 17), se observa que por la gran diferencia entre los precios de los productos que imperan en los países firmantes del TLC, la industria nacional se encuentra en desventaja.

En el Estado de Chihuahua existen además otros problemas que son relevantes y que afectan al sector forestal (SPE, 1999):

C Existe una desigual distribución de los recursos forestales entre los diferentes poseedores privados y sociales, lo que ha provocado una lucha constante sobre el recurso, con intereses particulares y de grupo que frecuentemente se ven enfrascados en litigios interminables.

C El bosque en general está en franco proceso de deterioro, en virtud de la progresiva explotación irracional a que está sometido; a la falta de cultivo y manejo integral; al desigual aprovechamiento de las especies que lo integran y de las partes del árbol que se utilizan.

C La tecnología que se aplica en todas las etapas de proceso, es de un atraso considerable en comparación con los países altamente productores y no se promueven suficientemente otras actividades económicas que contribuyan a agregar valor a la materia prima, o a vigilar y mejorar los recursos asociados como el agua, la flora y fauna (biodiversidad).

Existen otros factores negativos: escasa infraestructura oficial y asistencia técnica; escasa infraestructura caminera; incendios forestales, etc.

Para aliviar estos problemas, el gobierno de Chihuahua en colaboración con los productores forestales ha planteado una serie de acciones, (SPE,1999):

C Fomento y protección de los recursos naturales en estrecha coordinación con el gobierno federal y los sectores social y privado.

C Gestión, generación y dirección de recursos económicos para la protección y fomento al medio forestal por parte de los diferentes niveles de gobierno.

C Fortalecimiento y aumento de la inversión forestal privada incluyendo la introducción de tecnologías industriales limpias.

En consonancia con estos objetivos, se plantean algunos lineamientos estratégicos (SPE,1999):

C Fortalecimiento de la vigilancia forestal, la prevención y combate de incendios, la sanidad del bosque, los programas de reforestación y producción de plantas, apoyos financieros, programas de capacitación y cultura forestal, organización de los productores y resolución de problemas agrarios.

C Apoyo al Consejo Técnico Consultivo Forestal y de Suelos para vincular los organismos encargados de la planeación y ejecución de los programas de ordenamiento territorial, conservación, aprovechamiento, manejo y desarrollo. 
C Impulso a la formación de cadenas productivas en el sector mueblero otras industrias que utilicen la madera como insumo.

C Apoyo al Fideicomiso Chihuahua Forestal, así como al Programa de Desarrollo Forestal (PRODEFOR) para la ejecución del Programa de Desarrollo Forestal Sustentable del Estado.

\section{COMENTARIOS FINALES}

A diferencia del panorama poco alentador que presenta el sector forestal nacional, el del estado de Chihuahua ofrece perspectivas más halagadoras. Esto se pone de manifiesto en la contribución al producto interno bruto que tiene el sector en la entidad, con respecto al PIB nacional y si se analiza el caso particular de esta industria, la de la madera y derivados es más importante para el estado que con respecto al total nacional.

El estado debe aprovechar el capital con que cuenta para darle un fuerte impulso a su industria forestal, como lo son: sus existencias maderables, su ubicación geográfica y la infraestructura en comunicaciones. Un impulso importante sería el contar con un laboratorio de investigación, certificación de productos maderables y capacitación, que le permitiera a los productores situar sus productos en los mercados que por lo pronto el TLC ha abierto, a un mejor precio $y$ en cumplimiento con las nuevas normas de calidad que rigen el mercado internacional.

Tabla 15. Saldo de la balanza comercial: exportaciones menos importaciones (miles de dólares 1993 - 1994 - 1995)

\begin{tabular}{||l|c|c|c||}
\hline \hline PRODUCTO & \multicolumn{3}{|c||}{ ANOS } \\
\cline { 2 - 4 } FORESTAL & 1993 & 1994 & 1995 \\
\hline Madera aserrada & $-70,734$ & $-85,203$ & 18,236 \\
\hline Tableros de madera & $-122,030$ & $-110,531$ & $-12,319$ \\
\hline Listones y molduras & 24,108 & 25,243 & 48,838 \\
\hline Celulosa y papel & $-846,839$ & $-1,245,703$ & $-1,211,686$ \\
\hline Otros & $-43,802$ & 882 & $-6,298$ \\
\hline Total & $-1,059,297$ & $-1,415,312$ & $-1,163,229$ \\
\hline
\end{tabular}

Fuente: CNIF, 1996.

Tabla 16. Comparación de precios de los productos forestales entre EUA y México

\begin{tabular}{||l|c|c|c|c||}
\hline \multirow{2}{*}{ PRODUCTO } & \multicolumn{4}{|c|}{ PRECIOS } \\
\cline { 2 - 5 } & México & EUA & $\begin{array}{c}\text { Razón México/EUA } \\
(\%)\end{array}$ & Ref. \\
\hline Tabla conífera lab. & 286 & 172 & 166 & 1 \\
\hline Tabla no conífera & 662 & 570 & 116 & 1 \\
\hline Cajas de empaque & 343 & 255 & 134 & 1 \\
\hline Molduras & 836 & 624 & 134 & 1 \\
\hline Tabla conífera Michoacán & 220 & 189 & 116 & 2 \\
\hline Tabla de caoba & 1401 & 720 & 195 & 3 \\
\hline
\end{tabular}

Ref. 1 y 3 en dólares por $\mathrm{m}^{3}$; 2 en dólares por millar de pies tabla

Fuentes: U.S. Department of Commerce. Harmonized Tariff of the USA, 1990; M. Hicks: Wood

Products, 1991; Información directa; INFOTEC, 1988, citado en Chapela, 1996) 
Tabla 17. Aranceles actuales y plazos de desgravación de fracciones arancelarias seleccionadas de los productos forestales ante el Tratado de Libre Comercio

\begin{tabular}{||l|l|l|l|l||}
\hline FRACCION & CONCEPTO & SGP $^{*}$ & PLAZO & ARANCEL \% \\
\hline 440200 & Carbón de madera & & c & 10 \\
\hline 440320 & Madera en rollo de coníferas & & c & 10 \\
\hline 440333401 & Madera en rollo de caoba & & c & 10 \\
\hline 44071002 & Tablas de coníferas & & cq & 10 \\
\hline 44072301 & Tablas de caoba & & c & 15 \\
\hline 44079101 & Tablas de encino & & cq & 15 \\
\hline 44081001 & Hojas de conífera para chapas & & & \\
\hline 440910 & Molduras & sí & a & 20 \\
\hline 441010 & Aglomerados de madera & sí & b & 20 \\
\hline 44121101 & $\begin{array}{l}\text { Triplay con chapa de madera } \\
\text { tropical }\end{array}$ & & c & 20 \\
\hline 44121901 & Triplay con madera de coníferas & sí & c & 15 \\
\hline 44140001 & Marcos de madera & sí & b & 20 \\
\hline 44160005 & Duelas & sí & b & 20 \\
\hline 44182001 & Puertas y umbrales & sí & b & 20 \\
\hline 4419 & Artículos de mesa & sí & b & 20 \\
\hline 44219099 & Otras manufacturas (muebles) & & c & 20 \\
\hline 47010001 & Pasta mecánica de madera & & d & 0 \\
\hline 470311 & $\begin{array}{l}\text { Pasta química de madera a la } \\
\text { sosa }\end{array}$ & & d & 0 \\
\hline 4801 & Bobinas de papel prensa & & bp & 15 \\
\hline \hline
\end{tabular}

Claves

B Plazo de desgravación 5 años

C Plazo de desgravación 10 años, (largo)

$\mathrm{Cq} \quad$ Plazo de desgravación 15 años, (extralargo)

$\mathrm{p}$ Requiere permiso previo de importación

* Sistema General de Preferencias

Fuentes: Tratado trilateral de Libre Comercio entre México, Canadá y Estados Unidos. Octubre, 1992 (citado en Chapela, 1996). 


\section{REFERENCIAS}

Alvarez-Icaza Longoria, P. y J.L. Viveros Salinas. 1997. La construcción del modelo mexicano de plantaciones forestales. Un acercamiento ambiental. Cuadernos Agrarios No.14:25-33.

Cámara Nacional de la Industria Forestal. 1991. Memoria económica 1991-1992. CNIF.

Cámara Nacional de la Industria Forestal. 1996. Memoria económica 1994-1995. CNIF.

Chapela y Mendoza, G. 1996. Panorama del sector forestal en México. Gaceta ecológica INE-SEMARNAP Nueva época, No.38:27-39.

Gobierno del Estado de Chihuahua. 1997. Información estadística No.17, Octubre.

Ignacio, E., P. Sappanen y G. Trevizo. 1997. Plantaciones forestales en México: Una alternativa para el manejo sustentable de los recursos naturales. Cuadernos Agrarios No.14:34-40.
INEGI. 1993. Anuario estadístico del estado de Chihuahua. INEGI, Gobierno del estado de Chihuahua. 408 p.

INEGI. 1993. Anuario estadístico del estado de Durango. INEGI, Gobierno del estado de Durango. $366 \mathrm{p}$.

Ruelas M., L. La industria de la impregnación en México. (sin publicar).

Secretaría de Agricultura y Recursos Hidráulico. 1994. Inventario Nacional Forestal Periódico 1992-1994. SARH, Subsecretaría Forestal y de la Fauna Silvestre. $81 \mathrm{p}$.

Secretaría del Medio Ambiente, Recursos Naturales y Pesca. 1999. Regionalización de los servicios técnicos forestales. Delegación Federal de la SEMARNAP en Chihuahua. Junio de 1999

Secretaría de Planeación y Evaluación. 1999. Plan Estatal de Desarrollo 19992004. Ediciones del Gobierno del Estado de Chihuahua. Chihuahua, Chih. 